\title{
Microbiote et système nerveux
}

\author{
J.-M. Lecerf \\ (C) Lavoisier SAS 2018
}

Voilà deux géants qui se rencontrent, a priori pour le meilleur. Deux alliés insoupçonnés qui œuvrent en synergie. Pour le nutritionniste mais aussi aujourd'hui pour tout médecin, le microbiote est la révélation des 15 dernières années. D'un tube, le système digestif est devenu le siège d'un incroyable réseau de connexions et d'interrelations entre l'environnement et le «milieu intérieur ». Alors que notre maigre connaissance se résumait jusqu'ici à un vague lieu de passage et de réabsorption de l'eau, et que la biologie s'arrêtait à la coprologie fonctionnelle et aux coprocultures, aujourd'hui, ce domaine explose. Il révolutionne la physiologie en mettant en jeu les multiples interactions neurohormonales, immunitaires, métaboliques entre le microbiote et son hôte.

Nous devons revoir notre copie.

En même temps, cette connaissance, possible grâce à l'expérimentation animale notamment, ouvre des horizons considérables sur la physiopathologie de tant de troubles pour lesquels aucune hypothèse n'était avancée : fibromyalgie, troubles envahissants du comportement (autisme), syndrome d'hyperactivité, déficit de l'attention, maladie d'Alzheimer, dépression, syndrome du côlon irritable ne sont pas sans lien avec une dysbiose et/ou une hyperperméabilité intestinale, le tout assorti d'une neuro-inflammation ; et la liste n'est pas close. Sans compter les pathologies métaboliques où le microbiote a déjà fait ses preuves : obésité, stéatose hépatique...

Plusieurs questions découlent de ces découvertes, et de nombreux écueils sont à notre portée. Qui a commencé ? Nos contemporains sont prompts à dénoncer des coupables faciles, lait et blé (caséine et gluten) en premier lieu. Ils ne sont probablement que victimes d'une hyperperméabilité intestinale laissant passer des molécules diverses : le point de départ pourrait être un stress (extrêmement précoce anté- ou néonatal) ; il est d'ailleurs surprenant de voir, chez l'animal, les conséquences de la séparation maternelle sur le microbiote. La dysbiose pourrait résulter de perturbations également très précoces du microbiote (du fait d'une césarienne, d'un allaitement artificiel ou d'une antibiothérapie appuyée). La dysbiose favorise le passage de molécules bactériennes qui stimulent l'inflammation et activent l'axe hypothalamo-hypophyso-surrénalien et entretiennent le stress. D'autres xénobiotiques ou certaines molécules issues du métabolisme digestif de certains composants de nos aliments pourraient « profiter » de cette hyperperméabilité intestinale pour parvenir au cerveau. Ainsi, face à des facteurs exogènes, nous ne sommes pas égaux en raison des spécificités de notre génétique mais aussi de notre microbiote et de l'interaction entre les deux.

Les écueils seraient de penser avoir atteint le Graal, car aujourd'hui, la simple administration de probiotiques (lesquels ?) est très décevante chez l'homme. Il va falloir encore chercher d'autres leviers d'action, voire de prévention pour ces pathologies mal connues et donc mal aimées. L'erreur serait aussi de mettre tous nos œufs dans le même panier et d'occulter les autres déterminants et les diverses facettes de ces maladies qui sont pour une part de plus en plus psychoneurosomatiques : dans le bon sens du terme, c'est-à-dire celui du lien entre le soma et le système nerveux... par l'intermédiaire d'un étranger qui nous est cher et proche : le microbiote. 